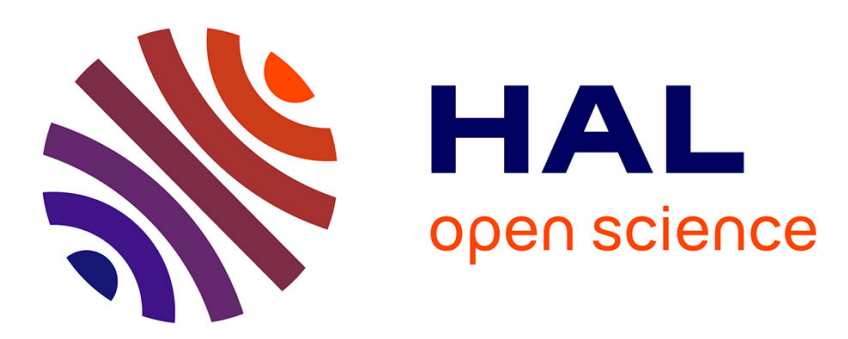

\title{
Using Plug\&Play Control for stable ACC-CACC system transitions
}

\author{
Francisco Navas, Vicente Milanés, Fawzi Nashashibi
}

\section{To cite this version:}

Francisco Navas, Vicente Milanés, Fawzi Nashashibi. Using Plug\&Play Control for stable ACCCACC system transitions. Intelligent Vehicles Symposium 2016, Jun 2016, Gothemburg, Sweden. 10.1109/IVS.2016.7535464 . hal-01304542

\section{HAL Id: hal-01304542 \\ https://inria.hal.science/hal-01304542}

Submitted on 19 Apr 2016

HAL is a multi-disciplinary open access archive for the deposit and dissemination of scientific research documents, whether they are published or not. The documents may come from teaching and research institutions in France or abroad, or from public or private research centers.
L'archive ouverte pluridisciplinaire HAL, est destinée au dépôt et à la diffusion de documents scientifiques de niveau recherche, publiés ou non, émanant des établissements d'enseignement et de recherche français ou étrangers, des laboratoires publics ou privés. 


\title{
Using Plug\&Play Control for stable ACC-CACC system transitions
}

\author{
F. Navas, V. Milanés and F. Nashashibi.
}

\begin{abstract}
This paper examines the already commercially available Adaptive Cruise Controller (ACC) system, and its evolution by adding vehicle-to-vehicle communications: the cooperative ACC (CACC) version. The transition between ACC and CACC controllers will be done through the new control technique called Plug\&Play. This technique is able to deal with living systems and the changes in its sensors and actuators to preserve the system stable. The aim is to ensure the system stability during transitions between controllers when the vehicle-to-vehicle communication link is changing from unavailable to available or vice versa.
\end{abstract}

\section{INTRODUCTION}

Road transport has significantly increased in recent years. According to the European Commission, road transport constitutes $82.4 \%$ of the whole transport for passengers in the European Union (EU) [1]. This situation points out an increment in the number of road vehicles, leading to a traffic congestion problem.

The intelligent transportation systems (ITS) domain aims to provide a more efficient solution to this kind of problems by developing aiding systems that helps drivers. Longitudinal speed control is a suitable aiding system to solve the traffic congestion problem in highways. Nowadays, the longitudinal speed control commercially available is the Adaptive Cruise Control (ACC) system, which allows the vehicle to keep a safety distance with a preceding vehicle by using a radar/laser front sensor [2]. Current research work explores the idea of adding vehicle-to-vehicle (V2V) communication to the already existing ACC systems, obtaining the socalled cooperative ACC systems (CACC). CACC will reduce the response time to speed changes, forming tighter stable vehicle strings [3].

The control structure for ACC relies on a frontal laser and a spacing policy [4]. A specific controller is designed to regulate the error between them, adjusting the speed to maintain a headway distance between vehicles in the same lane. The availability of a new sensor into the control structure, such V2V communication, will make necessary the re-design of the ACC-Controller.

Most of the controllers tend to be designed at the time of manufacturing and often in a dedicated microprocessor that does not allow modifications. Consequently, if new components or subsystems are added to the control loop, the design needs to be re-done, dealing with the high engineering and manufacturing costs. When new parts of the system become available for use in the control system, it is desirable

\footnotetext{
*Authors are with the Robotics and Intelligent Transportation Systems (RITS) Team, Inria Paris, 56 Rue du Charolais, 75012 Paris, France \{francisco.navas-matos, vicente.milanes, fawzi.nashashibi\}@inria.fr
}

to keep the original control than decommissioning the entire existing control [5].

The idea of adding new parts to an existing system online is known as Plug\&Play control theory [6]. It is an advanced control system that avoids the constant redesign necessary in systems with frequent instrumentation changes. The challenge comes with the definition of a control algorithm that can automatically accommodate this kind of changes while preserving the stability. The Plug\&Play control technique has been used in different practical applications such as control of a buffer tank [6], a laboratory district heating system [7], a live-stock stable [8], a fluid process [9], a supermarket refrigeration system [10] and an aerospace launch vehicle [11].

Now, one intends to apply this new control technique to the ITS framework. The quantity of changes that could affect a vehicle and its control law is large. In this paper, a specific application for accommodating the communication into the CACC using Plug\&Play control will be developed.

\section{Problem Formulation}

The principal objective of a CACC system is to form vehicles in a string as tightly as possible to decrease the traffic congestion problem. For achieving such goal, a wireless communication link is used. For the interest of this control study, the communication will be seen as a "device" added to the control loop. Current factory ACC algorithms have not been designed having in mind the possibility of adding cooperative behaviors in the near-future. Therefore, the idea is to be able to add the new control law to the existing controller when the communication becomes available, while leaving the existing control in place. In this way, it is not necessary to change the existing controller, preserving always the stability of the system.

On that purpose, the basis of the called Plug\&Play Control, which is able to handle changes in control living systems, will be explained. The basis is the Youla-Kucera parameterization [12]. This parameterization allows to know all the controllers that stabilize a given plant. This specific structure will be used for ensuring stable transitions.

The identification process of the vehicle will be done too. From this model, the design of two robust controllers for both ACC and CACC systems will be carried out. Later, Plug\&Play control theory will be used to ensure the stability during transitions between both controllers, transforming them using Youla-Kucera Parameterization.

\section{A. Plug\&Play Control}

The basis of Plug\&Play control is briefly described here, introducing some concepts such double coprime factorization 


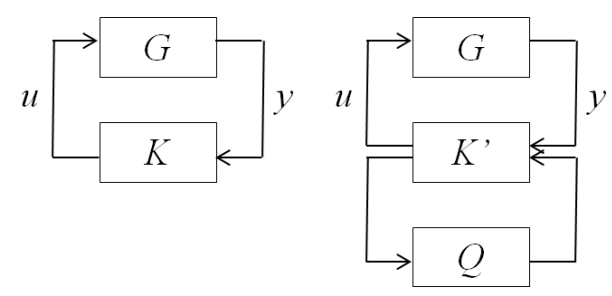

Fig. 1: Interconnection of a system $G$ and the controller $K$ (left). A different stabilizing controller implemented through the Youla-Kucera parameter $Q$ (right).

and Youla-Kucera parameterization of all stabilizing controllers (see [13], [14], [15] and [16] for further details).

Firstly, the scheme in the left in Fig. 1 is considered. $u$ and $y$ are controllable input and measurable output respectively. $G$ is the model of the plant under consideration and $K$ the initial controller able to stabilize the system $G$. That will be the initial situation, where an ACC controller is already installed in the vehicle.

According to [17], to proceed with the Youla-Kucera parameterization, the model $G$ should be factorized as the product of two different coprime factors, $N$ and $M$ or $N^{\prime}$ and $M^{\prime}$. These factors should have no pole-zero cancellations:

$$
G=N M^{-1}=M^{\prime-1} N^{\prime}
$$

The controller $\mathrm{K}$ is factorized with the coprime factors $U$, $V, U^{\prime}$ and $V^{\prime}$ :

$$
K=U V^{-1}=V^{\prime-1} U^{\prime}
$$

The resulting eight factors should be chosen to fulfill the double Bezout identity, as follows:

$$
\begin{array}{r}
{\left[\begin{array}{cc}
V^{\prime} & -U^{\prime} \\
-N^{\prime} & M^{\prime}
\end{array}\right]\left[\begin{array}{ll}
M & U \\
N & V
\end{array}\right]=} \\
=\left[\begin{array}{cc}
M & U \\
N & V
\end{array}\right]\left[\begin{array}{cc}
V^{\prime} & -U^{\prime} \\
-N^{\prime} & M^{\prime}
\end{array}\right]=\left[\begin{array}{ll}
1 & 0 \\
0 & 1
\end{array}\right]
\end{array}
$$

This factorization allows to obtain the parameterization of all controllers that stabilize a given plant $G$ :

$$
\begin{aligned}
K^{\prime} & =(U+M Q)(V+N Q)^{-1}= \\
& =\left(V^{\prime}+Q N^{\prime}\right)^{-1}\left(U^{\prime}+Q M^{\prime}\right)
\end{aligned}
$$

where $Q$ is a stable transfer function of appropriate dimensions called Youla-Kucera parameter.

Hence, based in an initial controller $K$ any other stable controller $K^{\prime}$ can be obtained through a stable parameter $Q$ (right part of fig. 1), in a smooth fashion and without losing stability. Thus, the initial ACC controller implemented in the vehicle will be changed to the CACC when the communication is available through $Q$.

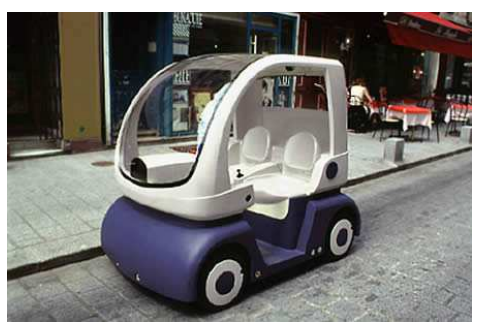

Fig. 2: Cycab robot.

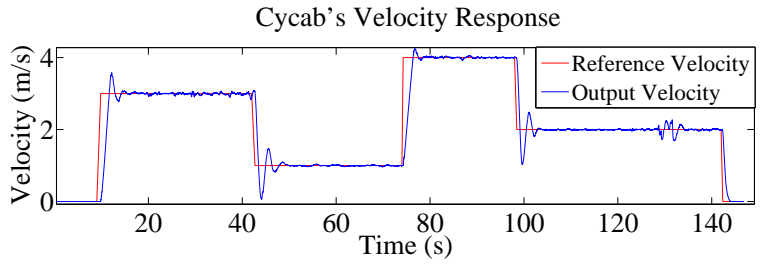

Fig. 3: Cycabs velocity response with velocity steps as reference.

\section{B. Longitudinal Dynamics Model}

Here, the longitudinal velocity model is obtained through identification techniques provided by MATLAB.

The vehicle to consider is a Cycab robot (see fig. 2). The Cycab is a mobile platform used in several research labs conceived for urban applications. From a kinematic point of view, it is important to highlight its principal difference with a classical vehicle: the Cycab turns its rear wheels as a linear function of the steering angle of the front wheels [18]. The vehicle is commanded through velocity. That velocity is limited to $4 \mathrm{~m} / \mathrm{s}$, because it is mainly designed for crowded areas where higher speeds in automated mode can lead to unsafe situations.

The Cycab robot is commanded with velocity steps within the limits in fig. 3 . The real velocity results have a characteristic overshoot that needs to be preserved in the model with the lowest order possible.

The model obtained is presented in (5). Fig. 4 shows how the characteristic overshoot is well-included in this model.

$$
G(s)=\frac{1}{0.2733 s^{2}+0.3228 s+1}
$$

\section{Adaptive CRUise Control}

ACC is an intelligent form of cruise control that slows down and speeds up automatically to keep a specific interdistance with the preceding vehicle. In our case, a string composed by three Cycab robots will be carried out.

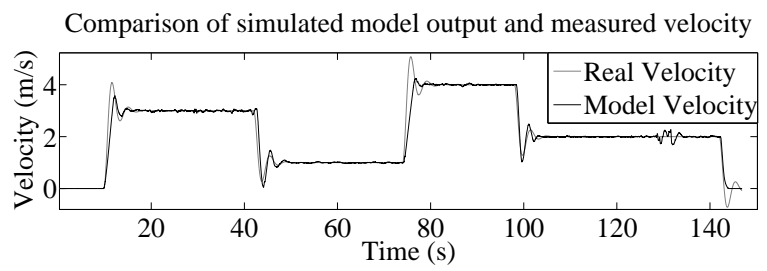

Fig. 4: Comparison between model output and real data. 
The desired distance between Cycab robots will be regulated by a constant head time spacing policy [19]. It will be done in function of the velocity of the vehicle $v_{i}$ and the constant head time $h_{d, i}$ :

$$
d_{i}=h_{d, i} v_{i}
$$

The control law is in charge of minimizing the error $e_{i}$ between the real distance laser-measured $d_{r, i}$ and the desired distance coming from the spacing policy $d_{i}$. Thus, the local control objective can be defined as the error regulation to zero, as follows:

$$
e_{i}=r_{r, i}-d_{i}
$$

This controller should ensure string stability. It can be defined as the attenuation of disturbances along the vehicle string, and it is evaluated through the string stability transfer function in (8), which takes into account the amplification of absolute position between two consecutive vehicles ( $Q i$ and $Q_{i-1}$ ) [20]. The maximum amplification of absolute position along the string can be interpreted through the H-infinity norm of the string stability transfer function (see (9)).

$$
\begin{gathered}
S S_{Q_{i}}=\frac{Q_{i}(s)}{Q_{i-1}(s)}, i \geq 1 \\
\left\|S S_{Q_{i}}\right\|_{\infty} \leq 1, \forall \omega, i \geq 1
\end{gathered}
$$

The chosen controller is a Proportional-Derivative (PD), to be able to predict the behavior of the error and to act in consequence. It will be designed through classical techniques, obtaining a stable controller able to ensure string stability too. It emulates the initial controller that could be in any ACC-equipped commercial vehicle.

Finally, the same controller will be parameterized through the coprime factorization, to be used in the Youla-Kucera controller modification in following sections, allowing stable transitions between ACC/CACC systems when communication is available.

\section{A. Classical Controller}

To regulate the error between the actual and the desired distances, a PD controller is used. Its output provides the target acceleration $u_{i}$ that is applied to the longitudinal dynamics model in (5) with and integrator (so $G(s)$ is reformulated in (11) ). Fig. 5 shows the ACC classical scheme. The PD controller structure is given in the Laplace domain as follows:

$$
U_{i}(s)=\left(\omega_{c, i}^{2}+\omega_{c, i} s\right) E_{i}(s)
$$

where $\omega_{c, i}$ is the bandwidth of the controller; The characteristic constants $k_{p}$ and $k_{d}$ are chosen in function of $\omega_{c, i}$ [21] to ensure easily comfort and non-saturation condition ( $\omega_{c, i}<<\omega_{q, i}$, where $\omega_{q, i}$ is the bandwidth of the vehicle).

$$
G(s)=\frac{V_{i}(s)}{U_{i}(s)}=\frac{1}{s\left(0.2733 s^{2}+0.3228 s+1\right)}
$$

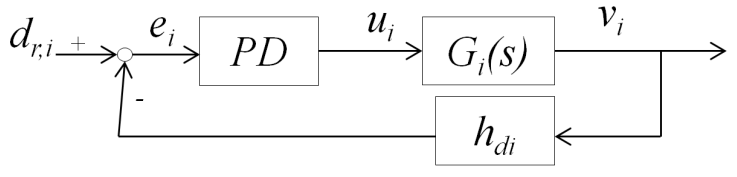

Fig. 5: ACC Classical Scheme.

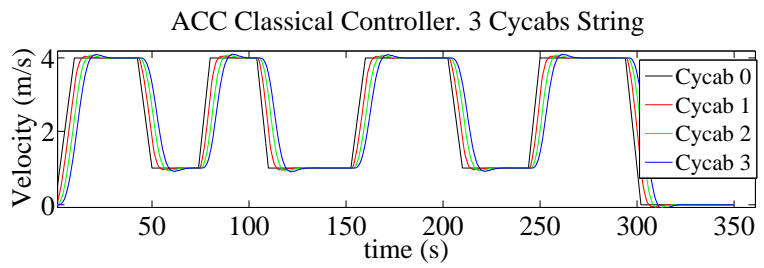

Fig. 6: Velocity results for a string of three Cycab robots equipped with the designed ACC. Head time $2 s$.

The limits of stability of the PD controller are defined through Rough-Hurwitz. The stability is ensured if:

$$
\begin{array}{r}
h_{d, i} \omega_{c, i}>0 \\
-0.895 \omega_{c, i}^{2}+\omega_{c, i} h_{d, i}+1>0 \\
\omega_{c, i}<\omega_{g, i}=2.7682 \mathrm{rad} / \mathrm{s}
\end{array}
$$

Stability analysis from (12) shows how the behavior of the system depends on the head time $h_{d, i}$ and the bandwidth of the controller $\omega_{c, i}$. For a specific $h_{d, i}$, the lower $\omega_{c, i}$ the higher overshoot and error, but without oscillations. On the contrary, the higher $\omega_{c, i}$ the lower overshoot and error, but with more oscillations present in the system. Therefore, $\omega_{c, i}$ should be chosen to have the lower oscillations and error possible, with the proper overshoot. An average value within the limits was chosen to balance both issues.

According to [19], the limits of string stability are obtained with (8) and (9) together with a simplified vehicle model $G_{i}(s)=s^{-2}$. The equation shown in (13), allows to obtain the minimum head time achievable $h_{d, i, \min }$ with a specific $\omega_{c, i}$. To enhance the congestion problem, the head time has to be as low as possible to increase the traffic capacity.

$$
h_{d, i}<h_{d, i, \min }=\sqrt{2} \omega_{c, i}^{-1}, i>1
$$

The minimum head time reachable results $h_{d, i, \text { min }}=2 \mathrm{~s}$ approximately, with $\omega_{c, i}=0.75 \mathrm{rad} / \mathrm{s}$. The velocity signal shown in fig. 6 validates the calculated head time to ensure string stability, since there is not amplification of the signal. One can appreciate how an imaginary vehicle 0 is used as a reference of the first vehicle in the string.

The design of this classical PD controller will be used as initial controller, emulating a factory ACC system.

\section{B. Coprime Factorization}

Up to this point, our attention must be drawn in the coprime factorization of the initial classical controller to be used in the Youla-Kucera controller modification, which allows us to introduce a new controller in the loop keeping the original one, when the communication is available.

The coprime factorization should be done obeying the Bezout identity too. On that purpose, in [17], a general 
method based in state space realization of the whole system is described. Two different steps need to be accomplished:

- "Translation" of the classical controller into the equivalent observer-based state feedback controller. Any classical controller, can be implemented as an observer based state controller knowing the closed loop poles of the system [22]. The plant should be observable and controllable.

- Apply the corresponding matricial equations to obtain the coprime factors.

The observed state feedback will control the plant through a lineal combination of the different states of the system with the gain matrix $F$. A Luenberger observer through a gain matrix $L$ will provide the states. It is known that availability of all the states of the system is unrealistic due to economical or physical problems. Both matrices, $F$ and $L$, need to be found. It will be possible only if the state space realization $(A, B, C, D)$ of the vehicle's model $G(s)$ is observable and controllable, that is, $\operatorname{rank}\left(C \quad C A C A^{2}\right)^{\prime}=n$ and $\operatorname{rank}\left(\begin{array}{lll}B & A B & A^{2} B\end{array}\right)=n$ respectively. $n$ is the number of states of $G(s)(n=3)$. Both conditions above are validated.

Considering the model $G(s)$ and an initial PD controller $K_{0}(s)$, it is easy to calculate the closed-loop response to a unit step reference. The closed-loop polynomial is the denominator of the following transfer function:

$$
C L(s)=\frac{K_{0}(s) G(s)}{1+K_{0}(s) G(s) h_{d, i, \min }}
$$

Once the closed loop poles are known, the pole placement design function provided by MATLAB is used to compute $F$ and $L$.

The classical controller can now be translated. The statespace realization of the system $G(s)$ along with the characteristic gains of the state observer $F$ and $L$ allow us to get the different coprime factors $N_{0}, M_{0}, U_{0}, V_{0}, N_{0}^{\prime}, M_{0}^{\prime}, U_{0}^{\prime}$ and $V_{0}^{\prime}$ for the initial controller. This is achieved thanks to the application of the following state spaces realizations:

$$
\begin{aligned}
& {\left[\begin{array}{cc}
M_{0} & U_{0} \\
N_{0} & V_{0}
\end{array}\right]=\left[\begin{array}{c|c:c}
A+B F & B & -L \\
\hline F & I & 0 \\
\hdashline C+D F & D & I
\end{array}\right]} \\
& {\left[\begin{array}{cc}
V_{0}^{\prime} & -U_{0}^{\prime} \\
-N_{0}^{\prime} & M_{0}^{\prime}
\end{array}\right]=\left[\begin{array}{c|c:c}
A+L C & -(B+L D) & L \\
\hline F & I & 0 \\
\hdashline C^{-} & -D^{-\cdots} & I
\end{array}\right]}
\end{aligned}
$$

Dashed lines indicated the matrices that belong to each factor.

Finally, the initial ACC controller is factorized in (17) and (18). The classical controller can be replaced by the factors $U_{0}$ and $V_{0}$ as shown in Fig. 7. The obtained factors fulfills the relation shown in (2).

$$
\begin{aligned}
U_{0} & =\frac{(s+0.75)}{(s+0.467)\left(s^{2}+0.7142 s+8.815\right)} \\
V_{0} & =\frac{1}{(s+0.467)\left(s^{2}+0.7142 s+8.815\right)}
\end{aligned}
$$

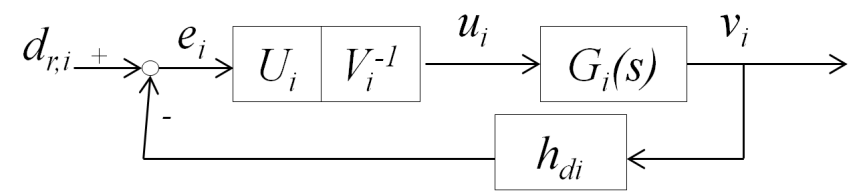

Fig. 7: ACC equivalent controller scheme obtained through coprime factorization

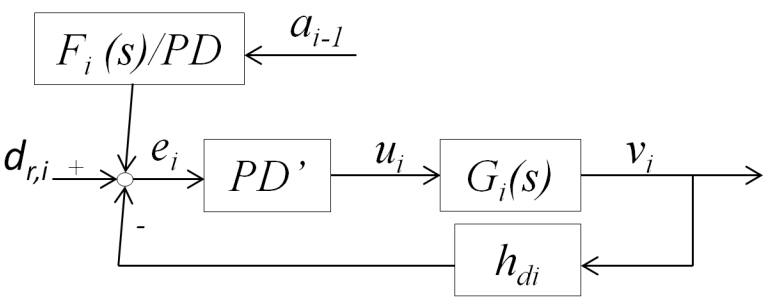

Fig. 8: CACC classical scheme.

\section{Cooperative Adaptive Cruise Control}

The cooperative version of ACC system includes wireless communication with its predecessor. Concretely, the acceleration $a_{i-1}$ will be sent to enhance the traffic congestion problem, reducing the minimum head time $h_{d, i, \min }$ that can be implemented.

It will be shown how the communication makes change the control structure, making useless the initial tuning of the PD controller. A new controller will be tuned in, obtaining the coprime factorization to be used as final controller in the Youla-Kucera controller modification.

\section{A. Classical Controller}

At this moment, the ACC system is extended to the cooperative version; adding the part corresponding to the wireless communication in fig. 8 to the control structure in fig. 5. Notice how the acceleration of the predecessor will be the input of a feedforward filter $F_{i}(s)$, whose transfer function in (19) helps to improve the string stability [23].

$$
F_{i}(s)=\frac{1}{H_{i}(s) G_{i}(s) s^{2}}, i>1
$$

For the cooperative case, the stability limits in (12) holds, as well as the use of the absolute position to study the string stability of the string composed by three Cycabs. If $F_{i}(s)$ is substituted into the string stability transfer function results in:

$$
S S_{Q_{i}}(s)=\frac{1}{1+h_{d, i} s}, i>1
$$

In this case the string stability is guaranteed for any positive value of head time, improving the congestion problem (see [24] for further details). The minimum head time reachable will be higher than zero due to the communication delay present in any wireless network. The chosen head time results $h_{d, i}=0.75 s$.

The addition of the communication to the initial PD controller with $\omega_{c, i}=0.75 \mathrm{rad} / \mathrm{s}$ causes instability. One can see in fig. 9 how the velocity response is oscillating when 
CACC Controller. Initial PD Configuration.

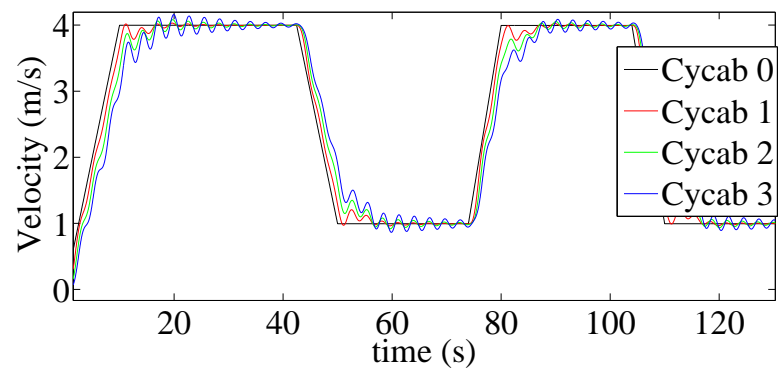

Fig. 9: Velocity results for a string of three Cycab robots equipped with CACC. Initial PD configuration. Head time $0.75 s$.

CACC Controller. Final PD Configuration.

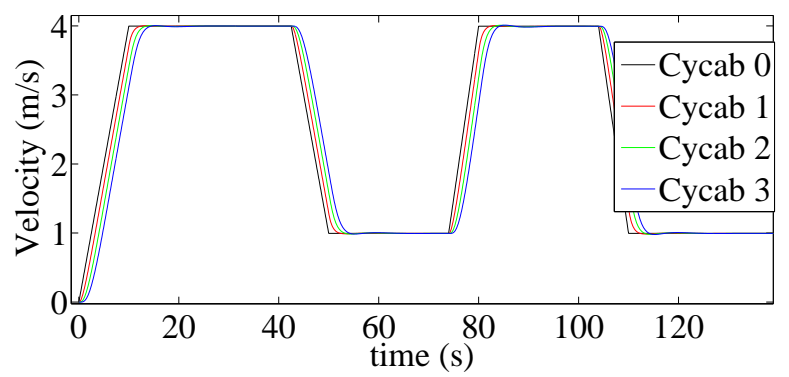

Fig. 10: Velocity results for a string of three Cycab robots equipped with CACC. Final PD configuration. Head time $0.75 \mathrm{~s}$.

the head time equals to $h_{d, i}=0.75 \mathrm{~s}$. These oscillations can make the system unstable in the transition ACC-CACC and affects to the fulfillment of the spacing policy. In order to prevent the situation the controller bandwidth is decreased to $\omega_{c, i}=0.6 \mathrm{rad} / \mathrm{s}$. Fig. 10 shows how the oscillations are removed. This is the final PD controller to be changed online when communication is available.

\section{B. Coprime Factorization}

Here, the final PD controller is factorized through the observer-based state feedback translation and the state space realization in (15) and (16), obtaining the coprime factors $U_{1}, V_{1}, N_{1}, M_{1}, U_{1}^{\prime}, V_{1}^{\prime}, N_{1}^{\prime}$ and $M_{1}^{\prime}$. The former controller can be replaced by the factors $U_{1}$ and $V_{1}$ as shown in fig. 11. It fulfills the factorization relation shown in (2).

$$
\begin{aligned}
U_{1} & =\frac{(s+0.6)}{(s+0.1932)\left(s^{2}+0.988 s+5.115\right)} \\
V_{1} & =\frac{1}{(s+0.1932)\left(s^{2}+0.988 s+5.115\right)}
\end{aligned}
$$

\section{Online Controller Modification}

Two different PD controllers have been designed for ACC and CACC systems respectively. Assuming that the ACC controller will always be in place, we now draw our attention to include the new PD controller through the Youla-Kucera parameter $Q$ when communication is available. The new

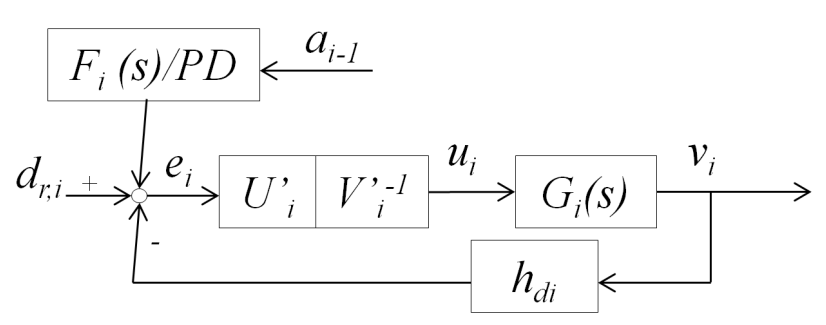

Fig. 11: CACC equivalent controller scheme obtained through coprime factorization.

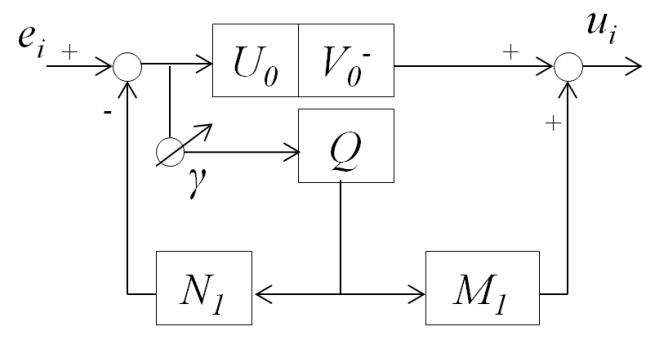

Fig. 12: Controller parameterization modified through $Q$ for connection to terminals of an existing controller.

controller will be included accessing only the terminals of the existing controller. The Youla-Kucera parameterization allows us to obtain all the controllers that stabilize a given plant $G$ through (4). Now, if the final stable controller $K_{1}$ and the initial $K_{0}$ are already known, the problem can be formulated inversely. Lets find $Q$ that makes the system changes from $K_{0}$ to $K_{1}$ :

$$
Q=U_{1}^{\prime}-V_{1}^{\prime} V_{0}^{\prime-1} U_{0}^{\prime}
$$

Substituting all the coprime factors $Q$ results:

$Q=\frac{(s+7.174)(s+4.146)(s+1.8)\left(s^{2}+37.95 s+393.9\right)}{0.0098(s+15)^{3}(s+20.12)\left(s^{2}+24.88 s+179.9\right)}$

The obtained YK parameter needs to be stable to ensure the stability of the final controller. This condition is achieved.

The control scheme to change from the initial controller to the final one is shown in fig. 12. Here, one can see how the change can be easily managed by modifying the value of $\gamma$ to 0 or 1 . Any lineal combination of $Q$ and $\gamma$ provides stable responses. The lineal combination of $Q$ and $\gamma$ doesn't make the poles of $Q$ unstables, so stable transitions are ensured thanks to (4). To prove so, two different transitions between controllers will be carried out: a step, and a 10 seconds transition ramp.

The step will be imposed by communication availability. The change from ACC to CACC is done in the least favorable situation, when the system has the lower velocity. The correct formulation of the problem is proved through the results in fig. 13.

The results corresponding to a ramp of $10 s$ in $\gamma$ are shown in fig. 14. In that case the weight of each controller will change with the evolution of $\gamma$ in time. The smoother change makes the transition between controllers smoother too. 
Change ACC to CACC. Youla-kucera. Step

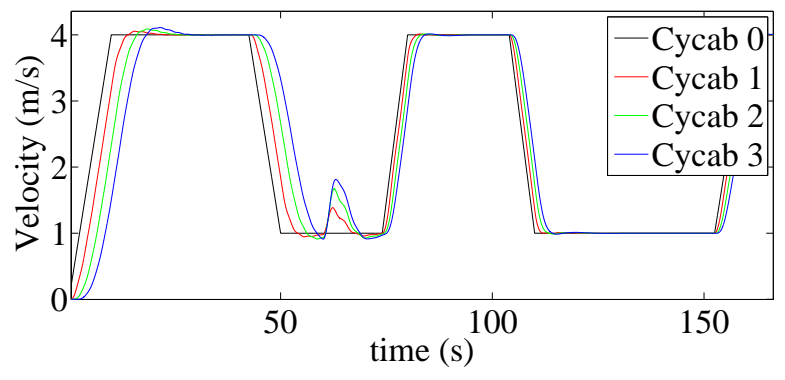

Fig. 13: Speed responses for an ACC-CACC transition using a step for controllers transition.

Change ACC to CACC. Youla-Kucera. Ramp

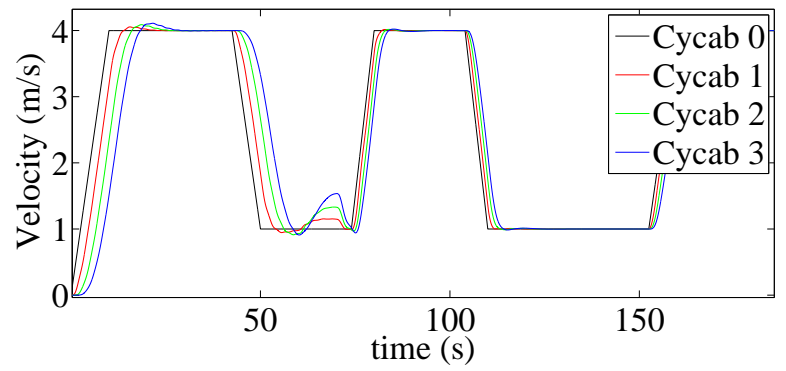

Fig. 14: Speed responses for an ACC-CACC transition using a ramp for controllers transition.

Experiments show the ability of Plug\&Play control to switch between controllers whereas keeping stability. It is also worth to mention that such transition can be done through a controllers switching or even by slightly modifying in the weight of each of them in the control action.

\section{CONCLUSiOnS AND Future WORKS}

Plug\&Play Control has been used into the ITS framework, concretely into the ACC-CACC change when V2V communication becomes available. The correct behavior of the system has been proved through MATLAB simulation results for a string composed by three Cycab robots.

The stability in transitions is ensured thanks to a stable Youla-Kucera parameter, guaranteeing system stability during transitions periods using Plug\&Play Control theory.

Furthermore, the existing controller of any commercial vehicle could be changed into another stable controller just by accessing the terminals of the original one. From an industrial point of view, it is a significant advantage because of the cost savings and the ability to switch back to an existing, proven control design if the system fails.

Future work will be focused on the experimental proof in three real Cycabs to validate the proposed approach. Additionally, current regulation of both controllers by YoulaKucera parameter $Q$ will be further investigated for improving vehicle stability in different scenarios.

\section{ACKNOWLEDGMENTS}

Authors express their gratitude to the CoCoVeA (Coopération Conducteur-Véhicule Automatisé) ANR project for its support in the development of this work.

\section{REFERENCES}

[1] E. Comission, EU transport in figures. Statistical pocketbook 20141, 2014.

[2] R. Jurgen, Adaptive Cruise Control. SAE International, 2006.

[3] V. Milanés and S. Shladover, "Cooperative adaptive cruise control in real traffic situations," IEEE Transactions on Intelligent Transportation Systems, vol. 15, no. 1, pp. 296-305, 2014

[4] L. Hong and G. Lilong, "Study on adaptive cruise control spacing policy and stability analysis," in International Conference on Electric Information and Control Engineering, 2011, pp. 5364-5367.

[5] A. Michelsen and R. Izadi-Zamanabadi, "Towards automatic model based controller design for reconfigurable plants," in IFAC World Congr., Seoul, Korea, 2008.

[6] J. Stoustrup, "Plug \& play: Control technology towards new challenges," European Journal of Control, pp. 311-330, 2009.

[7] K. Trangbaek and T. Knudsen, "Plug and play process control of a district heating system," in European Journal of Control, 2006, pp. $4084-4089$

[8] K. Trangbaek and J. Bendtsen, "Stable controller reconfiguration through terminal connections a practical example," in 7th IEEE Int. Conf. Control Autom., 2009, pp. 2037-2042.

[9] S. Bodenburg, "Plug-and-play control theory and implementation," in IEEE International Conference on Industrial Informatics, 2013, pp. $165-170$.

[10] A. Michelsen and J. Stoustrup, "Model based plug and play process control of a supermarket refrigeration system: A heuristic approach," in European Control Conference, 2009, pp. 4090-4095.

[11] L. Jin-Yong and Z. Shuo, "Study on the architecture of plug-and-play control system for aerospace launch vehicle," in Third International Conference on Instrumentation, Measurement, Computer, Communication and Control, 2013, pp. 1488-1493.

[12] V. Kucera, "A method to teach the parameterization of all stabilizing controllers," In Proc. 18th IFAC World Congress, vol. 18, no. 1, p. $6355,2011$.

[13] H. Niemann, "Parameterisation of extended systems," IEEE Proc.: Control Theory Appl., vol. 153, pp. 221-227, 2006.

[14] V. Kucera, "Stability of discrete linear feedback systems," in 6th IFAC World Congr., 1976.

[15] D. C. Youla and H. A. Jabr, "Modern wiener-hopf design of optimal controllers part ii: The multivariable case," IEEE Trans. Autom. Control, vol. 21, no. 3, p. 319338, 1976.

[16] B. D. O. Anderson, "From youla-kucera to identification, adaptive and nonlinear control," Automatica, vol. 34, p. 14851506, 1998.

[17] J. Bendsten and K. Trangbaek, "Plug-and-play control modifying control systems online," IEEE Trans. Control Systems Technology, vol. 21, no. 1, pp. 79-93, 2013.

[18] S. Sekhavat and J. Hermosillo, "The cycab robot: a differentially flat system," in IEEE/RS International Conference on Intelligent Robots and Systems, 2000.

[19] G. Naus and R. Vugts, "Cooperative adaptive cruise control and design and experiments," in American Control Conference, 2010.

[20] M. Khatir and E. Davison, "Decentralized control of a large platoon of vehicles using nonidentical controllers," in In Proc. Amer. Control Conf., 2003, p. 27692776.

[21] J. Ploeg and B. Scheepers, "Design and experimental evaluation of cooperative adaptice cruise control," in In Proc. IEEE Intell. Transp. Syst. Conf., 2011, p. 260265.

[22] J. I. Yuz, "From classical to state-feedback-based controllers," IEEE Control Systems, vol. 23, pp. 58-67, 2003.

[23] J. Naus and P. Vugts, "String-stable cacc design and experimental validation: A frequency-domain approach," IEEE Transactions on vehicular technology, vol. 59, p. 42684279, 2010.

[24] S. Oncu and J. Ploeg, "Cooperative adaptive cruise control networkaware analysis of string stability," IEEE on Intelligent Transportation Systems, vol. 15, pp. 1527-1537, 2014. 